\title{
Effects of dialectical behavior therapy skills training on outcomes for mental health staff in a child and adolescent residential setting
}

\author{
Ann F. Haynos*1, Alan E. Fruzzetti ${ }^{2}$, Calli Anderson ${ }^{3}$, David Briggs ${ }^{3}$, Jason Walenta ${ }^{3}$ \\ ${ }^{1}$ University of Minnesota Medical Center, United States \\ ${ }^{2}$ University of Nevada, Reno, United States \\ ${ }^{3}$ Willow Springs Center, United States
}

Received: October 12, 2015

Accepted: December 8, 2015

Online Published: January 5, 2016

DOI: $10.5430 /$ jha.v5n2p55

URL: http://dx.doi.org/10.5430/jha.v5n2p55

\begin{abstract}
Training in Dialectical Behavior Therapy (DBT) skills coaching is desirable for staff in psychiatric settings, due to the efficacy of DBT in treating difficult patient populations. In such settings, training resources are typically limited, and staff turnover is high, necessitating brief training. This study evaluated the effects of a brief training in DBT skills coaching for nursing staff working in a child and adolescent psychiatric residential program. Nursing staff $(n=22)$ completed assessments of DBT skill knowledge, burnout, and stigma towards patients with borderline personality disorder (BPD) before and after a six-week DBT skills coaching training. Repeated measure ANOVAs were conducted to examine changes on all measures from pre- to post- treatment and hierarchical linear regressions to examine relationships between pre- training DBT knowledge, burnout, and BPD stigma and these same measures post-training. The brief DBT skill coaching training significantly increased DBT knowledge $(p=.007)$ and decreased staff personal $(p=.02)$ and work $(p=.03)$ burnout and stigma towards BPD patients $(p=.02)$. Burnout indices and BPD stigma were highly correlated at both time points $(p<.001)$; however, while pre-training BPD stigma significantly predicted post-training client burnout $(p=.04)$, pre-training burnout did not predict post-training BPD stigma. These findings suggest that brief training of psychiatric nursing staff in DBT skills and coaching techniques can result in significant benefits, including reduced staff burnout and stigma toward patients with BPD-related problems, and that reducing BPD stigma may particularly promote lower burnout.
\end{abstract}

Key Words: Dialectical behavior therapy, Training, Implementation, Psychiatric residential treatment, Nursing staff

\section{INTRODUCTION}

Translation of empirically supported psychological treatment practices into a pre-existing mental health system is a task that is often challenging, and too frequently unsuccessful. It is widely recognized that a substantial gap exists between evidence-based practices delivered to fidelity and the stan- dard of practice in most mental health systems. ${ }^{[1]}$ Barriers frequently cited to explain this implementation gap include lack of staff knowledge and skill in delivering empirically supported treatments, limited administrative resources dedicated to staff training, and inadequate leadership and infrastructural support dedicated to developing evidence based

\footnotetext{
*Correspondence: Ann F. Haynos; Email: afhaynos@ umn.edu; Address: Department of Psychiatry, University of Minnesota, 2450 Riverside Ave., F279, Minneapolis, MN 55454, United States.
} 
practices. ${ }^{[1-3]}$

Dialectical Behavior Therapy (DBT) is a psychological treatment with a considerable evidence base for treating severe and multi-problem patients. ${ }^{[4]}$ DBT was originally developed for adults with borderline personality disorder (BPD) and related problems. However, since its inception, DBT has been demonstrated to be effective in reducing a wide range of problems, including suicide ideation and attempt, non-suicidal self-injury, depression, bulimic symptoms, and substance abuse, across a variety of patient populations. ${ }^{[5]}$ Recently, DBT has also been applied successfully to treating multi-problem adolescents and, in this population, DBT has been found to reduce suicidal ideation and behavior, nonsuicidal self-injury, psychiatric symptoms, and treatment utilization. ${ }^{[6]}$

Because DBT has been demonstrated to be effective for treating a variety of difficult problems across patient populations, the demand for DBT training of mental health professionals has substantially increased. ${ }^{[7]}$ The extant literature on DBT training indicates that typical training methods, such as comprehensive workshops and internet-based training programs, are associated with increases in DBT skill knowledge and utilization among mental health professionals, and that individuals with widely varying levels of education and training can acquire competency in DBT under expert training and supervision. ${ }^{[3,8-10]}$ Research further indicates that clinicians who have received DBT-informed training report lower professional burnout, less stigma towards individuals with personality disorders, and more optimistic attitudes towards treating BPD clients. ${ }^{[11,12,14]}$ Thus, there is evidence that training in DBT methods is effective and beneficial when delivered to mental health professionals working with severe and multi-problem clients.

The research on DBT training and implementation to date has been conducted primarily with masters-level clinicians delivering DBT individual therapy and skills training groups. However, little information is available regarding effective means of training nursing staff (e.g., mental health technicians, psychiatric nurses) working in inpatient or residential psychiatric settings. There are many institutional barriers to training residential line staff in DBT techniques, including extremely limited time allotted for training and high turnover for staff in these positions. ${ }^{[15]}$ Moreover, while a consultation team, which aims to improve skill in implementing DBT techniques, while simultaneously decreasing provider burnout, is a regular part of any DBT program for therapists and skill training leaders, ${ }^{[4]}$ nursing staff rarely enjoy the benefits of a such a meeting. However, in inpatient or residential settings in which DBT is delivered, nursing staff can play a vital role in implementation, as these staff generally have the most frequent patient contact and are thereby the most available for coaching in DBT skill generalization. Therefore, even when comprehensive DBT training of nursing staff is not feasible, some amount of training in DBT skills and coaching techniques is likely to be advisable. In particular, brief training of nursing staff that results in rapid knowledge and skill acquisition, while conferring the benefits associated with DBT training, is desirable. In order to garner institutional support for DBT training and implementation for staff in these roles, clear demonstration of benefits for both nursing staff and patients may be needed. However, currently little research exists on brief and effective means of training line staff in DBT techniques.

Therefore, in the current study, the authors evaluated the effects of a brief training that focused on teaching DBT skills and skill coaching techniques to mental health nursing staff working in a child and adolescent residential psychiatric treatment facility. Based on the findings from prior DBT training and implementation research, the authors hypothesized that the brief DBT skills coaching training for nursing staff would result in significant: 1) Increases in DBT skill knowledge; 2) Decreases in staff burnout; and 3) Decreases in stigma towards individuals with BPD. The authors were also interested in the interplay between DBT skill knowledge, burnout, and stigma towards individuals with BPD, as this information could help to effectively tailor future DBT-oriented training programs for psychiatric staff. For instance, if two or more of these variables were highly interrelated, future interventions could potentially be streamlined by targeting one process in order to produce an effect in the other related variables. Such information would be especially useful if one process were found to be particularly predictive of positive outcomes and, therefore, targeting of this process could be expected to of greatest benefit in the training of nursing staff. Therefore, the authors also conducted exploratory analysis to examine the cross-sectional and longitudinal associations between DBT skill knowledge, burnout, and BPD stigma among mental health staff.

\section{METHODS}

\subsection{Participants}

Participants comprised three cohorts of mental health nursing staff, consisting of primarily mental health technicians and nurses, who completed DBT skill and skill coaching training between October 2011 and March $2013(n=22)$. Training completion was defined as attending at least 8 out of 12 training hours. Some of the staff attending the training worked exclusively on a residential unit dedicated to delivery of comprehensive DBT treatment, while others worked on 
units in which DBT skill coaching was not an explicit focus. Due to the nature of staffing in the facility, staff members from different units were included in the training, primarily in order to improve their DBT coaching for occasions when they were temporarily assigned to the DBT unit. None of the staff participating in the training had previously received formal training in DBT theory or techniques. Due to the sensitive nature of work- and client- related questions, and relatively small sample size, demographics were not collected on training participants in order to protect staff anonymity. An appropriately convened institutional review board approved all aspects of this research.

\subsection{Treatment facility}

Training was conducted at a 116-bed residential facility specializing in psychiatric treatment of children and adolescents ages five to seventeen. The facility contains separate units designed to treat varying age levels and psychiatric presentations, including a unit exclusively dedicated to DBT treatment for adolescents. On the DBT treatment unit, adolescents received weekly DBT individual and family sessions and daily skill training groups, as well as other DBT-informed therapeutic groups. Nursing staff, particularly mental health technicians, were the individuals primarily responsible for in vivo coaching in effective use of DBT skills in order to promote generalization of the skills that the adolescents had learned in group and individual therapy sessions.

\subsection{Training procedures}

The DBT skills coaching training consisted of six two-hour interactive training sessions delivered over twelve weeks, which focused on acquisition of DBT skills knowledge and strategies for implementing effective skill coaching. The DBT skills coaching training sessions all contained the following structural components: 1) Review of the homework assignment from the prior training session, which consisted of an assignment to practice coaching patients in the DBT skill techniques highlighted in the previous session. Homework review allowed opportunity for reinforcement of effective skill coaching delivery, as well as provision of feedback and problem-solving regarding any difficulties encountered in skill coaching; 2) Instruction in new DBT skills and practice coaching in new skill techniques using role plays; 3) Assignment of coaching homework to be practiced before the subsequent training. A detailed list of the DBT skills taught in each session is listed in Table 1. The DBT skills coaching training was delivered by a Ph.D. level clinical psychologist who is an internationally recognized expert trainer in DBT with more than 20 years experience in delivery, training, and supervision of DBT. The expert trainer was assisted by two intensively-trained master's level (in marriage and family therapy and clinical psychology, respectively) DBT clinicians, both of which had at least two years experience delivering DBT.

Table 1. Description of the skills content presented in the brief DBT skills coaching training for nursing staff

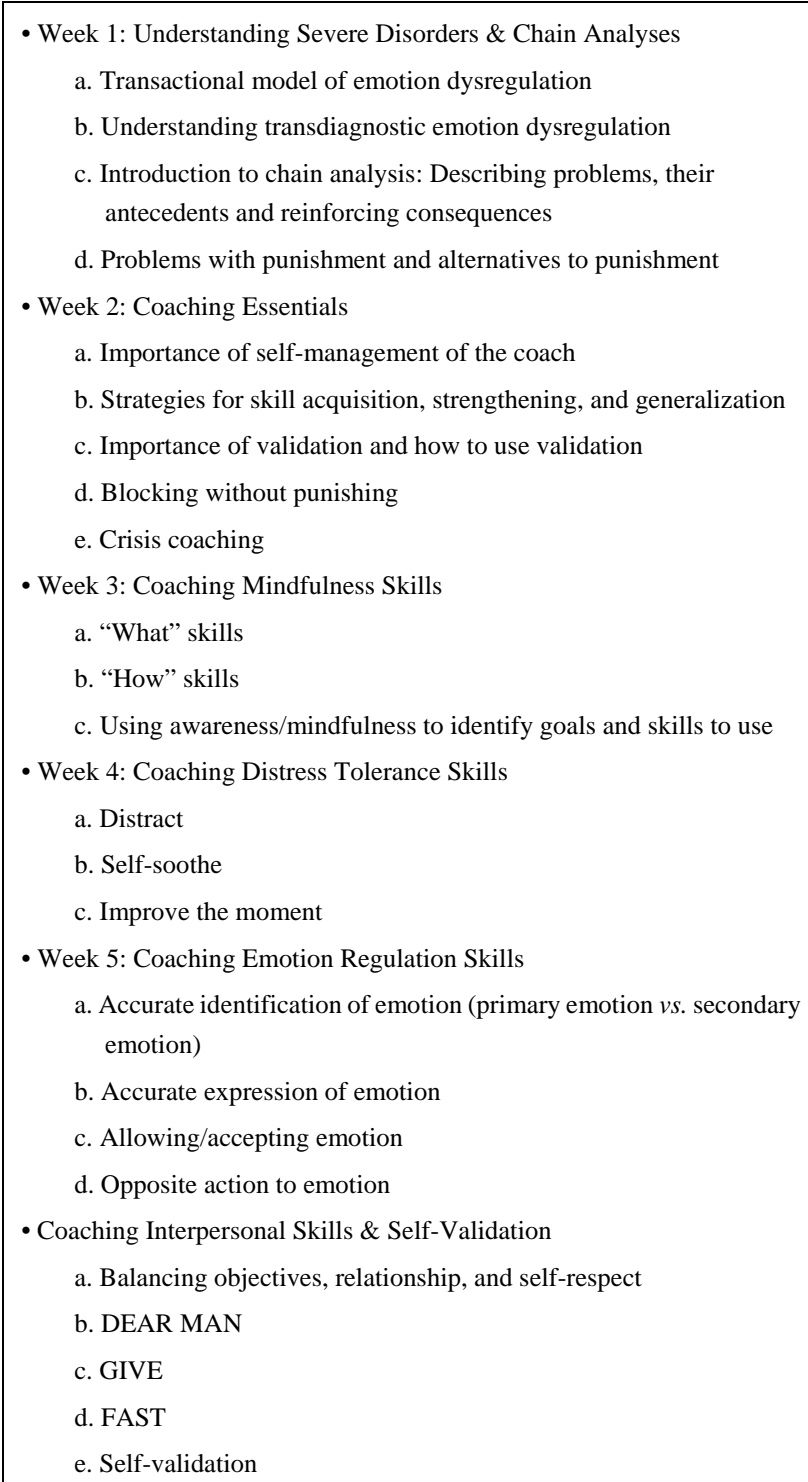

\subsection{Measures}

All measures were administered prior to the first and following the last training session. A ten-item DBT skill knowledge test was used to measure acquisition of the DBT skills taught throughout the training. This measure was adapted from a knowledge test frequently used in DBT intensive clinical training (K. Comtois, personal communication, October 2, 2011), though adjusted slightly to correspond with the content of the training described in this study. Similar knowledge tests have been previously used in DBT training research, with each adapted to match the specific content of the train- 
ing being tested. ${ }^{[8-10]}$ The test consisted of multiple choice or matching questions pertaining to the content taught in the skills training, each of which had one correct answer out of four potential options. Scores on this test were calculated as percentage of questions answered correct; therefore, potential scores ranged from 0-100, with higher scores representing greater DBT skill knowledge. Though the research team was unable to collect test-retest reliability of this measure at each time point, baseline and post-training scores on the DBT skill knowledge test were moderately correlated $(r=.42, p=.04)$, providing some evidence consistent with adequate test-retest reliability.

The Copenhagen Burnout Inventory $(\mathrm{CBI})^{[16]}$ was used to assess burnout. This is a nineteen-item measure used to assess different facets of burnout, including the following: 1) Personal burnout: burnout related to physical and psychological exhaustion (six items); 2) Work-related burnout: burnout related to general aspects of the work environment (seven items); and 3) Client-related burnout: burnout related specifically to interacting with clients (six items). Scores on each of these subscales range from 0-100, with higher scores representing a great degree of burnout. The CBI has been found to have adequate reliability and construct validity. ${ }^{[16]}$ In this sample, the internal consistency of the CBI subscales, as measured by Cronbach's alpha ranged from .82 to .91 at baseline and from .74 to .87 following the training.

The Attitudes toward BPD Questionnaire (ABPDQ) ${ }^{[17]}$ was used to assess stigma towards individuals with a diagnosis of BPD and related problems. The ABPDQ is a thirty-item scale, in which each item describes a stigmatizing statement towards individuals with BPD (or a non-stigmatizing statement in the case of reverse-scored items) and responses are scored on a 5-point Likert scale ranging from 1 = Disagree Strongly to $5=$ Agree Strongly. Thus, scores on this scale range from 30 to 150 , with higher scores indicating greater stigma towards individuals with BPD. In this sample, the internal consistency of the subscales, as measured by Cronbach's alpha was .88 at baseline and .90 following training.

\subsection{Analyses}

Repeated measures ANOVAs were conducted to examine changes in skill knowledge, burnout, and BPD stigma pre- to post-training. Cohen's d analyses were used to estimate effect size. ${ }^{[18]}$ Pearson's correlations were conducted between DBT skill knowledge, CBI scores, and ABPDQ scores at pre- and post- training assessment points in order to examine the relationship between these variables. Hierarchical linear regressions were conducted to further examine the relationship between DBT skill knowledge, staff burnout, and BPD stigma. Because of the small sample size, bootstrapping with 1,000 samples was used in all regression models. To examine predictors of each facet of post-training burnout, the pre-test CBI score for the facet being examined was entered into the first step, and pre-test DBT skill knowledge and ABPDQ scores were entered into the second step, of the regression. To examine predictors of post-training ABPDQ scores, pre-test ABPDQ scores were entered into the first step, and pre-test DBT skill knowledge and all three CPI scores were entered into the second step, of the regression. All predictors were centered to reduce problems related to multicollinearity.

\section{Results}

As highlighted in Table 2, there was a significant increase in DBT skill knowledge $(p=.007)$ and were significant decreases in the personal $(p=.03)$ and work $(p=.02)$ burnout subscales of the CBI, as well as a significant decrease in ABPDQ scores $(p=.02)$ from pre- to post-training. However, there were no significant reductions in the client burnout subscale of the CBI post-training.

Table 2. Means, standard deviations, and ANOVA results between pre- and post- training staff DBT skill knowledge, burnout, and BPD stigma scores

\begin{tabular}{|c|c|c|c|c|c|c|c|c|}
\hline & \multicolumn{2}{|c|}{ Pre-training } & \multicolumn{2}{|c|}{ Post-training } & \multirow{2}{*}{$\boldsymbol{F}$} & \multirow{2}{*}{$d f$} & \multirow{2}{*}{$p$} & \multirow{2}{*}{$d$} \\
\hline & $M(S D)$ & Range & $M(S D)$ & Range & & & & \\
\hline DBT knowledge (\% correct) & $63.27(13.80)$ & $38.50-92.30$ & 74.21 (17.68) & $33.30-92.30$ & 52.01 & 1.21 & $.007^{*}$ & 2.47 \\
\hline CBI personal burnout score & 42.05 (21.59) & $0.00-91.67$ & 34.52 (18.44) & 4.17-79.17 & 5.80 & 1.21 & $.03^{*}$ & 0.38 \\
\hline CBI work burnout score & $42.53(21.11)$ & 7.14-96.43 & 35.52 (18.06) & $7.14-75.00$ & 6.06 & 1.21 & $.02^{*}$ & 0.36 \\
\hline CBI client burnout score & 25.85 (17.69) & $0.00-58.33$ & $22.58(16.15)$ & $0.00-50.00$ & 0.93 & 1.21 & .35 & 0.19 \\
\hline ABPDQ score & 71.09 (14.33) & $45.00-95.00$ & $65.27(17.81)$ & $41.00-105.00$ & 6.26 & 1.21 & $.02^{*}$ & 0.36 \\
\hline
\end{tabular}

${ }^{*} p<.05$; DBT: Dialectical Behavior Therapy; CBI = Copenhagen Burnout Inventory; ABPDQ = Attitudes toward Borderline Personality Disorder Questionnaire

As highlighted in Table 3, scores on the ABPDQ and CBI personal, work, and client burnout subscales were all highly significantly correlated with each other at both pre- and post- training assessment points $(p<.001)$, suggesting that patient stigma and staff burnout to be highly related at both assessment points, such that more stigmatizing attitudes towards 
BPD were associated with greater burnout across domains. DBT skill knowledge was significantly negatively correlated with ABPDQ at pre-test $(p=.04)$, but not post-test $(p=.20)$, suggesting that those with greater knowledge of DBT before training were significantly less stigmatizing of BPD patients.

Table 3. Correlations between DBT skill knowledge, burnout, and BPD stigma scores Pre-training

\begin{tabular}{|c|c|c|c|c|c|c|}
\hline & Measure & 1 & 2 & 3 & 4 & 5 \\
\hline \multirow{5}{*}{ Pre-training } & 1. DBT skill knowledge & ----- & & & & \\
\hline & 2. CBI personal burnout & -.04 & ----- & & & \\
\hline & 3. CBI work burnout & -.08 & $.90^{* *}$ & ----- & & \\
\hline & 4. CBI client burnout & -.31 & $.63^{*}$ & $.75^{* *}$ & ----- & \\
\hline & 5. ABPBQ & $-.43^{*}$ & $.72^{* *}$ & $.70^{* *}$ & $.71^{* *}$ & ---- \\
\hline \multirow{5}{*}{ Post-training } & 1. DBT skill knowledge & ----- & & & & \\
\hline & 2. CBI personal burnout & -.03 & ----- & & & \\
\hline & 3. CBI work burnout & .16 & $.80^{* *}$ & ----- & & \\
\hline & 4. CBI client burnout & .01 & $.74^{* *}$ & $.80^{* *}$ & ----- & \\
\hline & 5. ABPBQ & -.29 & $.68^{* *}$ & $.62^{*}$ & $.79^{* *}$ & ---- \\
\hline
\end{tabular}

Controlling for pre-test CBI client burnout scores, the model including ABPDQ and DBT knowledge pre-test scores significantly predicted post-test client burnout, $F(2,18)=6.00$, $p=.01, R^{2}=0.28$. However, only ABPDQ significantly contributed to the model, $B=0.86, S E=0.30, p=.01$. Controlling for pre-test CBI scores, the model including ABPDQ and DBT knowledge did not predict personal, $F(2,18)=1.67, p=.22, R^{2}=0.07$, or work, $F(2,18)=1.30, p=.30, R^{2}=0.05$, burnout post-training. Controlling for pre-test BPD stigma, the model including CBI and DBT knowledge pre-test scores did not significant predict post-test ABPDQ scores, $F(4,16)=0.68, p=.62$, $R^{2}=0.05$.

\section{Discussion}

In this study, the authors examined the impact of a brief DBT skills coaching training for nursing staff in a child and adolescent residential psychiatric setting on DBT skill knowledge acquisition, burnout, and stigma towards individuals with BPD and related concerns. The results suggest that the brief DBT skills coaching training increased knowledge of DBT skills and reduced personal and work-related burnout, as well as patient stigma. These results support the utility of providing brief DBT skill coaching training for nursing staff in inpatient or residential psychiatric settings, at least in facilities providing treatment for children and adolescents.

This study adds to a growing literature suggesting that individuals with varied educational and training backgrounds are willing to engage in training in DBT techniques and that these trainings can result in the acquisition of DBT knowledge for such individuals. ${ }^{[3,8-10]}$ However, this is the first study demonstrating the ability to deliver DBT knowledge in

Published by Sciedu Press a brief and relatively low burden format to nursing staff working within a residential psychiatric setting. In such settings, staff turnover tends to be high and resources limited, ${ }^{[15]}$ making it unlikely that most staff members can access the more typical intensive or comprehensive DBT training. In this study, even just twelve hours of training was sufficient for producing significant DBT skill knowledge acquisition and salutary effects on staff burnout and stigma towards patients. Training of such brevity may be more feasible to deliver to nursing staff in inpatient and residential settings than more traditional training methods, and therefore may allow for increased delivery of evidence-based treatment practices across different levels of staff.

Further, decreasing staff burnout through DBT skills coaching training is of notable benefit to psychiatric staff, patients, and institutions. Effects of staff burnout include diminished emotional and physical health, reduced job satisfaction and performance, increased absenteeism and turnover, poorer continuity of care, greater institutional costs, increased negative attitudes towards patients, and, ultimately, poorer patient outcomes. ${ }^{[19]}$ Thus, any means of decreasing burnout among staff in a psychiatric setting is desirable. In line with other research, ${ }^{[12,15]}$ the findings of this study suggest that training in DBT techniques can assist in reducing destructive staff burnout levels. Further, the results of this study suggest that even a small amount of time dedicated to staff training in DBT skills can lead to significant declines in both personal and work burnout. Thus, there is potentially a substantial benefit to psychiatric residential institutions for implementing a low-burden intervention. Though the decreases in the client burnout subscale of the CBI were non-significant following training in this study, initial client burnout scores among the staff in this sample $(M=25.85)$ were below previously reported estimates for patient care workers $(M=30.9){ }^{; 20]}$ therefore, there may have been a floor effect for this variable. In contrast, the personal $(M=42.05)$ and work-related burnout $(M=42.53)$ scores in this sample were higher than those previously reported $\left(M=33.0\right.$ and 35.9, respectively). ${ }^{[20]}$ These discrepancies in the domains of burnout could reflect that the nursing staff were working within a high acuity setting, which may have resulted in elevated personal and work-related burnout, but several of the staff were already working within a unit guided by DBT principles, which may have resulted in decreased client-related burnout.

The significant decreases in BPD stigma following the DBT skill coaching training are also notable. Given that, consistent with the DBT model, ${ }^{[4]}$ the training included a focus on the importance of taking a non-judgmental perspective towards even the most difficult of patient behaviors and iden- 
tifying valid parts of patient experiences (such as primary emotions) to validate, this result was anticipated. Further, this finding is consistent with results of previous studies, which have found training in DBT techniques to reduce stigmatizing attitudes towards patients. ${ }^{[13,14]}$ This outcome is important, as individuals with BPD are a highly stigmatized group, with healthcare workers expressing more negative attitudes towards individuals with BPD than patients with other psychiatric problems. ${ }^{[14]}$ Perhaps unsurprisingly, perceived stigma has been found to contribute to poorer treatment outcomes for individuals with BPD. ${ }^{[21]}$ Therefore, an intervention that reduces stigma poses a potential benefit for patients on a residential unit. This is appealing for institutions that aim to improve patient outcomes.

Further, stigma against patients with BPD-related difficulties and personal, work, and client burnout were highly positively related at both time points. These findings are consistent with past research, which has found that stigmatization towards patients among mental health workers is associated with more self-stigma and poorer job satisfaction. ${ }^{[22]}$ Additionally, stigma towards BPD patients at baseline predicted client burnout following training, even after controlling for pretreatment burnout levels. In contrast, pre-treatment burnout did not significantly predict BPD stigma following training. This suggests that less stigma towards BPD patients is protective against developing additional burnout related to client interactions. This presents a potential pathway to promote reductions in staff burnout following DBT training; DBT training may reduce burnout by increasing understanding and compassion for BPD individuals through an explicit focus on being less judgmental toward patients and more validating of their experiences. This also suggests a motivator for staff members to reduce stigma towards difficult patients, as this appears to benefit staff as well as patients. One concern that could be raised is that a significant relationship between BPD stigma and burnout continued to exist even following the DBT skills coaching training. This could suggest that even further emphasis on increasing validation and decreasing judgment towards individuals with BPD may be important when training nursing staff in DBT skills coaching techniques.

A strength of this study is that the training was delivered in a brief format, thereby reducing time and resource burden. However, one issue for consideration is that all findings may be subject to a "dose response" effect of training that this study would not be able to detect: Perhaps more training would have continued to produce increasingly positive effects, and possibly less training would have some effect. The effect sizes in the present study are small to medium; therefore more training of nursing staff may be needed to produce larger effects over time. Further research should explore this, as well as begin to identify moderators of these training effects (e.g., staff factors that might influence outcomes). Additionally, training for this study was conducted primarily by an expert with extensive experience providing DBT training. It is unknown whether training by individuals with significantly less experience would achieve similar results. The skill training was also conducted in relatively small groups, was interactive, and was delivered over many weeks. This training format intentionally provided opportunities for staff to practice skills coaching both in the training in order to receive coaching and feedback themselves and between training sessions. Although other formats might be considered (e.g., 12 hours over two full days of training), it is unknown whether briefer or more consolidated training would yield the same results. Nevertheless, future studies should explore these options.

Another major strength of this study is that the training was conducted in an existing residential institution, which lends ecological validity to the results, suggesting brief DBT skill coaching training could be feasibly implemented in similar settings. However, there are also study limitations related to the conduct of research on an existing unit, which include small sample size, lack of control group, and inability to collect demographic information or test-retest reliability on measures. Though the findings were sufficiently robust to detect significant results even with a limited sample, it is possible that changes in burnout and stigma are better accounted for by time and/or experience, rather than specifically attributable to the training. This is unlikely, however, as prior research has found that burnout significantly increases and compassion for patients significantly decreases with greater time spent working in a psychiatric facility. ${ }^{[23]}$ Further, it was not possible to collect direct patient outcomes for this study, though anecdotal evidence suggests that staff training in DBT skill coaching techniques resulted in fewer unit shutdown and seclusion and restraint procedures. Finally, the study was conducted specifically in a child and adolescent residential facility. Therefore, it is unclear whether the findings of this study generalize to facilities providing for adult patients. Future evaluations of brief DBT skill coaching training for psychiatric nursing staff should include larger and more diverse samples, a comparison group to control for potential confounds, more internally valid study designs, and direct patient-related outcomes.

\section{Conclusions}

Brief training in DBT skills coaching for psychiatric nursing staff can result in significant benefits, including reduced staff burnout and stigma toward patients with BPD-related 
problems. Further, lower stigma towards multi-problem individuals predicts staff burnout over time, suggesting that this may be a particularly important training target among staff working in a psychiatric residential facility. These findings suggest that such training is a worthwhile investment for inpatient and residential facilities utilizing a DBT model that wish to improve outcomes for staff and patients.

\section{ACKNOWLEDGeMENTS}

Research reported in this publication was partially supported by a pre-doctoral training fellowship awarded by the National Institute of Mental Health under Award Number F31MH097450. The content is solely the responsibility of the authors and does not necessarily represent the official views of the National Institutes of Health.

\section{REFERENCES}

[1] Hanson RF, Gros KS, Davidson TM, et al. National trainers' perspectives on challenges to implementation of an empirically-supported mental health treatment. Adm Policy Ment Health. 2014 Jul; 41(4): 522-34. http://dx.doi.org/10.1007/s10488-013-0492-6

[2] Bond GR, Drake RE, McHugo GJ, et al. Long-term sustainability of evidence-based practices in community mental health agencies. Adm Policy Ment Health. 2014 Mar; 41(2): 228-36. http: //dx.doi.org/10.1007/s10488-012-0461-5

[3] Hawkins KA, Sinha R. Can line clinicians master the conceptual complexities of dialectical behavior therapy? An evaluation of a State Department of Mental Health training program. J Psychiatr Res. 1998 Nov-Dec; 32(6): 379-84. http://dx.doi.org/10.1016/S 0022-3956 (98) 00030-2

[4] Linehan MM. Cognitive-behavioral treatment of borderline personality disorder. New York: Guilford Press; 1993.

[5] Lynch TR, Trost WT, Salsman N, et al. Dialectical behavior therapy for borderline personality disorder. Annu Rev Clin Psychol. 2007 Apr; 3: 181-205. http://dx.doi.org/10.1146/annurev.cli npsy.2.022305.095229

[6] Klein DA, Miller AL. Dialectical behavior therapy for suicidal adolescents with borderline personality disorder. Child Adolesc Psychiatr Clin N Am. 2011 Apr; 20(2): 205-16. http://dx.doi.org/10. 1016/j.chc. 2011.01 .001

[7] Swenson CR, Torrey WC, Koerner K. Implementing dialectical behavior therapy. Psychiatr Serv. 2002 Feb; 53(2): 171-8. http: //dx.doi.org/10.1176/appi.ps.53.2.171

[8] Dimeff LA, Harned MS, Woodcock EA, et al. Investigating bang for your training buck: a randomized controlled trial comparing three methods of training clinicians in two core strategies of dialectical behavior therapy. Behav Ther. 2015 May; 46(3): 283-95. http://dx.doi.org/10.1016/j.beth.2015.01.001

[9] Dimeff LA, Koerner K, Woodcock EA, et al. Which training method works best? A randomized controlled trial comparing three methods of training clinicians in dialectical behavior therapy skills. Behav Res Ther. 2009 Nov; 47(11): 921-30. http://dx.doi.org/10.1016 /j.brat.2009.07.011

[10] Dimeff LA, Woodcock EA, Harned MS, et al. Can dialectical behavior therapy be learned in highly structured learning environments? Results from a randomized controlled dissemination trial. Behav Ther. 2011 Jun; 42(2): 263-75. http://dx.doi.org/10.1016/j .beth.2010.06.004

[11] Hazelton M, Rossiter R, Milner J. Managing the 'unmanageable': training staff in the use of dialectical behaviour therapy for borderline personality disorder. Contemp Nurse. 2006 Feb-Mar; 21(1): 120-30. http://dx.doi.org/10.5172/conu.2006.21.1.120

[12] Perseius KI, Kåver A, Ekdahl S, et al. Stress and burnout in psychiatric professionals when starting to use dialectical behavioural therapy in the work with young self-harming women showing borderline personality symptoms. J Psychiatr Ment Health Nurs. 2007 Oct; 14(7): 635-43. http://dx.doi.org/10.1111/j.1365-285 $0.2007 .01146 . \mathrm{x}$

[13] Clarke S, Taylor G, Bolderston H, et al. Ameliorating patient stigma amongst staff working with personality disorder: Randomized controlled trial of self-management versus skills training. Behav Cogn Psychother. 2015 Nov; 43(6): 692-704. http://dx.doi .org/10. $1017 / \mathrm{S} 1352465814000320$

[14] Knaak S, Szeto ACh, Fitch K, et al. Stigma towards borderline personality disorder: effectiveness and generalizability of an anti-stigma program for healthcare providers using a pre-post randomized design. Borderline Personal Disord Emot Dysregul. 2015 May; 2: 9. http://dx.doi.org/10.1186/s40479-015-0030-0

[15] Carmel A, Fruzzetti AE, Rose ML. Barriers and solutions to implementing Dialectical Behavior Therapy in a public behavioral health system. Community Ment Health J. 2014 Jan; 50(1): 25-30. http://dx.doi.org/10.1007/s10597-013-9679-2

[16] Kristensen TS, Borritza M, Villadsena E, et al. The Copenhagen Burnout Inventory: A new tool for the assessment of burnout. Work \& Stress. 2005 Feb; 19(3): 192-207. http://dx.doi.org/10.10 80/02678370500297720

[17] Fruzzetti AE. Attitudes toward borderline personality disorder questionnaire (ABPDQ). University of Nevada, Reno; 2004. PMid: 15669548.

[18] Cohen J. Statistical power analysis for the behavioral sciences (2nd ed.). Hillsdale, NJ: Lawrence Earlbaum Associates; 1998.

[19] Morse G, Salyers MP, Rollins AL, et al. Burnout in mental health services: a review of the problem and its remediation. Adm Policy Ment Health. 2012 Sep; 39(5): 341-52. http://dx.doi .org/10 1007/s10488-011-0352-1

[20] Borritz M, Rugulies R, Bjorner JB, et al. Burnout among employees in human service work: design and baseline findings of the PUMA study. Scand J Public Health. 2006 Feb; 34(1): 49-58. http://dx.doi.org/10.1080/14034940510032275

[21] Rüsch N, Schiel S, Corrigan PW, et al. Predictors of dropout from inpatient dialectical behavior therapy among women with borderline personality disorder. J Behav Ther Exp Psychiatry. 2008 Dec; 39(4): 497-503. http://dx.doi.org/10.1016/j.jbtep. 2007. 11.006

[22] Verhaeghe M, Bracke P. Associative stigma among mental health professionals: implications for professional and service user well-being J Health Soc Behav. 2012 Mar; 53(1): 17-32. http://dx.doi .org /10.1177/0022146512439453

[23] Rossi A1, Cetrano G, Pertile R, et al. Burnout, compassion fatigue, and compassion satisfaction among staff in community-based mental health services. Psychiatry Res. 2012 Dec 30; 200(2-3): 933-8. http://dx.doi.org/10.1016/j.psychres.2012.07.029 